\title{
Deformations of bimodule problems
}

\author{
by
}

Christof G e i ß (México, D.F.)

\begin{abstract}
We prove that deformations of tame Krull-Schmidt bimodule problems with trivial differential are again tame. Here we understand deformations via the structure constants of the projective realizations which may be considered as elements of a suitable variety. We also present some applications to the representation theory of vector space categories which are a special case of the above bimodule problems.
\end{abstract}

1. Introduction. Let $\mathrm{k}$ be an algebraically closed field. Consider the variety $\operatorname{alg}_{V}(\mathrm{k})$ of associative unitary k-algebra structures on a vector space $V$ together with the operation of $\mathrm{Gl}_{V}(\mathrm{k})$ by transport of structure. In this context we say that an algebra $\Lambda_{1}$ is a deformation of the algebra $\Lambda_{0}$ if the corresponding structures $\lambda_{1}, \lambda_{0}$ are elements of $\operatorname{alg}_{V}(\mathrm{k})$ and $\lambda_{0}$ lies in the closure of the $\mathrm{Gl}_{V}(\mathrm{k})$-orbit of $\lambda_{1}$. In [11] it was shown, using Drozd's tame-wild theorem, that deformations of tame algebras are tame.

Similar results may be expected for other classes of problems where Drozd's theorem is valid. In this paper we address the case of bimodule problems with trivial differential (in the sense of [4]); here we interpret deformations via the structure constants of the respective projective realizations. Note that the bimodule problems include as special cases the representation theory of finite-dimensional algebras $([4,2.2])$, subspace problems (4.1) and prinjective modules $([16,1])$. We also discuss some examples concerning subspace problems.

We understand that W. W. Crawley-Boevey has obtained similar results. P. Dräxler draw my attention to examples as in 4.3.

1991 Mathematics Subject Classification: 16G60, 16G20, 16E40.

Key words and phrases: bimodule problems, vector space categories, tame, wild, deformations, degenerations.

Part of this work was done while the author was supported by a catedra patrimonial of CONACYT at the Instituto de Matemáticas, UNAM (México). 


\section{Bimodule problems}

2.1. Let us recall some basic definitions from [4] and [18]. A category is called a k-category if all morphism spaces are k-modules and the composition is $\mathrm{k}$-bilinear; a functor between $\mathrm{k}$-categories is called a k-functor if it is $\mathrm{k}$-linear. As a general convention we will compose morphisms in k-categories from left to right.

A k-category is a Krull-Schmidt category if it has finite direct sums, split idempotents and the morphism spaces are all finite-dimensional. If $\mathcal{K}$ is a k-category, a $\mathcal{K}$ left- (resp. right-) module is a contravariant (resp. covariant) $\mathrm{k}$-functor $\mathcal{K} \rightarrow \mathrm{k}$-mod, and accordingly, a $\mathcal{K}$-bimodule is a $\mathrm{k}$ functor $\mathcal{K}^{\mathrm{op}} \times \mathcal{K} \rightarrow \mathrm{k}$-mod. If $M$ is a $\mathcal{K}$-bimodule, we write conveniently $a m b:=m M(a, b) \in \mathcal{K}\left(X^{\prime}, Y^{\prime}\right)$ for $m \in M(X, Y), a \in \mathcal{K}\left(X^{\prime}, X\right)$, and $b \in \mathcal{K}\left(Y, Y^{\prime}\right)$.

Definition. A Krull-Schmidt bimodule problem is a pair $(\mathcal{K}, M)$, where $\mathcal{K}$ is a Krull-Schmidt k-category and $M$ a $\mathcal{K}$-bimodule. The category $\operatorname{Mat}(\mathcal{K}, M)$ has as objects the pairs $(X, m)$ with $X \in \operatorname{Obj} \mathcal{K}, m \in M(X, X)$ and $\operatorname{Mat}(\mathcal{K}, M)\left((X, m),\left(X^{\prime}, m^{\prime}\right)\right):=\left\{f \in \mathcal{K}\left(X, X^{\prime}\right) \mid m f=f m^{\prime}\right\}$.

Since in the following we will consider only bimodule problems which are Krull-Schmidt, we sometimes drop the words "Krull-Schmidt" for brevity.

Re mark. The categories Mat $(\mathcal{K}, M)$ are Krull-Schmidt categories, being a special case of the categories considered in $[4,2]$.

2.2. Let $(\mathcal{K}, M)$ be a bimodule problem where $\mathcal{K}$ has only a finite number of isoclasses of indecomposable objects. It is of finite type if $\operatorname{Mat}(\mathcal{K}, M)$ admits only a finite number of isoclasses of indecomposable objects. Recall from [4] that the bimodule problem $(\mathcal{K}, M)$ may be formulated quite directly as normal free triangular linear bocs, and thus if $(\mathcal{K}, M)$ is not of finite type then it is either tame or wild by the theorem of Drozd.

For convenience let us trace back the definitions of tame and wild for bocses from [2] through the constructions in [4]. In order to formulate the result, we need however some setup - essentially we adapt the corresponding notions from [8]. Let $X \in \operatorname{Obj} \mathcal{K}, \mathbf{b}:=\left(b_{1}, \ldots, b_{n}\right)$ some k-base of $M(X, X)$ and $\varphi:=\left(\varphi_{1}, \ldots, \varphi_{n}\right)$ a sequence of elements of some k-algebra $R$. Then we call the triple $(X, \mathbf{b}, \varphi)$ an $R$-frame for $(\mathcal{K}, M)$. Such an $R$-frame induces a functor

$$
F_{X, \mathbf{b}, \varphi}: \bmod -R \rightarrow \operatorname{Mat}(\mathcal{K}, M), \quad N \mapsto\left(\left.X \otimes N\right|_{\mathrm{k}}, \eta\left(\sum_{i=1}^{n} b_{i} \otimes N\left(\varphi_{i}\right)\right)\right) .
$$

Here $X \otimes W$ represents the functor $\operatorname{Hom}_{\mathrm{k}}(W, \mathcal{K}(X, ?))$ (hence $X \otimes \mathrm{k}^{n} \cong X^{n}$, see $[8,2.1])$; since we deal only with finite-dimensional vector spaces there 
is a natural isomorphism $\eta: M(X, Y) \otimes \operatorname{Hom}_{\mathrm{k}}(V, W) \rightarrow M(X \otimes V, Y \otimes W)$. Finally, $N\left(\varphi_{i}\right) \in \operatorname{End}_{\mathrm{k}}\left(\left.N\right|_{\mathrm{k}}\right)$ is induced by the module multiplication $n \mapsto n \varphi_{i}$.

Definition. The bimodule problem $(\mathcal{K}, M)$ is wild if for some $X \in$ Obj $\mathcal{K}$ there exists a $\mathrm{k}\langle x, y\rangle$-frame $(X, \mathbf{b}, \boldsymbol{\varphi})$ such that the induced functor $F_{X, \mathbf{b}, \varphi}: \bmod -\mathrm{k}\langle x, y\rangle \rightarrow \operatorname{Mat}(\mathcal{K}, M)$ preserves indecomposability and respects isoclasses.

$(\mathcal{K}, M)$ is tame if for every $X \in \operatorname{Obj} \mathcal{K}$ there exists a finite number of $\mathrm{k}[t]$-frames $\left(X, \mathbf{b}^{(j)}, \varphi^{(j)}\right)$ such that for every indecomposable object $(X, m)$ in $\operatorname{Mat}(\mathcal{K}, M)$ we have $(X, m) \cong F_{\left(X, \mathbf{b}^{(j)}, \varphi^{(j)}\right)}(\mathrm{k}[t] /(t-\lambda))$ for some $j$ and some $\lambda \in \mathrm{k}$.

Remark. If $(\mathcal{K}, M)$ corresponds to a wild subspace problem (see 4.1), we can find by the results of [8] for some $X$ a k $\langle x, y\rangle$-frame $\left(\mathbf{b}=\left(b_{1}, \ldots, b_{n}\right)\right.$, $\boldsymbol{\varphi}=(1, x, y, 0, \ldots, 0))$ such that the induced functor preserves indecomposability and respects isoclasses.

2.3. Let $\Lambda$ be a finite-dimensional associative k-algebra and $M$ a finitedimensional $\Lambda$ - $\Lambda$-bimodule. Consider $\mathcal{P}(\Lambda)$, the category of finitely generated projective $\Lambda$-left modules, and the functor

$$
\bar{M}: \mathcal{P}(\Lambda)^{\mathrm{op}} \times \mathcal{P}(\Lambda) \rightarrow \mathrm{k}-\bmod , \quad\left(P_{1}, P_{2}\right) \mapsto \operatorname{Hom}_{\Lambda}\left(P_{1}, M \otimes_{\Lambda} P_{2}\right) .
$$

Then $(\mathcal{P}(\Lambda), \bar{M})$ is a Krull-Schmidt bimodule problem, where $\mathcal{P}(\Lambda)$ has a finite number of isoclasses of indecomposable objects.

Remark. Every bimodule problem $(\mathcal{K}, M)$ where $\mathcal{K}$ has only a finite number of isoclasses of indecomposable objects is of the above form. Indeed, let $\left\{X_{1}, \ldots, X_{n}\right\}$ represent the indecomposable objects of $\mathcal{K}$ and set $\Lambda_{\mathcal{K}}:=\bigoplus_{i, j=1}^{n} \mathcal{K}\left(X_{i}, X_{j}\right)$, which becomes a k-algebra with the obvious matrix multiplication; further, set $\underline{M}:=\bigoplus_{i, j=1}^{n} M\left(X_{i}, X_{j}\right)$ with the corresponding $\Lambda_{\mathcal{K}}-\Lambda_{\mathcal{K}}$-bimodule structure. Now it is not hard to see that $\operatorname{Mat}(\mathcal{K}, M)$ and $\operatorname{Mat}\left(\mathcal{P}\left(\Lambda_{\mathcal{K}}\right), \underline{\bar{M}}\right)$ are equivalent categories. We call the above construction the projective realization of $(\mathcal{K}, M)$ (compare [18, 2.5], [19, Sec. 17.9]).

2.4. For given vector spaces $V, W$ consider the affine variety $\operatorname{algb}_{V}^{W}(\mathrm{k})$ which consists of the pairs $(\lambda, \mu) \in \operatorname{Hom}_{\mathrm{k}}(V \otimes V, V) \times \operatorname{Hom}_{\mathrm{k}}(V \otimes W \otimes V, W)$, where $\lambda$ defines an associative unitary k-algebra structure $\Lambda$ on $V$, and $\mu$ defines a $\Lambda$-bimodule structure $M$ on $W$ (compare [7], [11]). On $\operatorname{algb}_{V}^{W}(\mathrm{k})$ operates the algebraic group $\mathrm{Gl}_{V, W}(\mathrm{k}):=\mathrm{Gl}_{V}(\mathrm{k}) \times \mathrm{Gl}_{W}(\mathrm{k})$ by transport of structure, i.e.

$(\lambda, \mu)^{g, h}\left(v_{1} \otimes v_{2}, v_{l} \otimes w \otimes v_{r}\right)=\left(\left(v_{1} g^{-1} \otimes v_{2} g^{-1}\right) \lambda g,\left(v_{l} g^{-1} \otimes w h^{-1} \otimes v_{r} g^{-1}\right) \mu g\right)$.

Definition. The bimodule problem $\left(\mathcal{K}_{1}, M_{1}\right)$ is a deformation of the problem $\left(\mathcal{K}_{0}, M_{0}\right)$ if for the respective projective realizations $\left(\mathcal{P}\left(\Lambda_{i}\right), \underline{\bar{M}}_{i}\right)$ the pairs $\left(\Lambda_{i}, \underline{M}_{i}\right)$ have the same underlying vector spaces $(V, W)$, and if for 
the corresponding structures $\left(\lambda_{i}, \mu_{i}\right) \in \operatorname{algb}_{V}^{W}(\mathrm{k})$ (always $\left.i \in\{0,1\}\right)$ we find $\left(\lambda_{0}, \mu_{0}\right)$ in the closure of the $\mathrm{Gl}_{V, W}(\mathrm{k})$-orbit of $\left(\lambda_{1}, \mu_{1}\right)$.

Theorem. Deformations of tame bimodule problems are tame.

The proof will be given in 3.6.

\section{Varieties}

3.1. Let $(\mathcal{K}, M)$ be a bimodule problem and $X \in \operatorname{Obj} \mathcal{K}$. Set mat $_{\mathcal{K}, M}^{X}(\mathrm{k})$ $:=M(X, X)$; it will be considered as an affine space in the sense of algebraic geometry. On $\operatorname{mat}_{\mathcal{K}, M}^{X}(\mathrm{k})$ operates the algebraic group $\operatorname{Aut}_{X}(\mathrm{k}):=$ $\{f \in \mathcal{K}(X, X) \mid f$ is invertible $\}$ by conjugation: $m^{f}:=f^{-1} m f$. Thus the orbits of $\operatorname{Aut}_{X}(\mathrm{k})$ on mat $_{\mathcal{K}, M}^{X}(\mathrm{k})$ correspond bijectively to the isoclasses of representations of $(\mathcal{K}, M)$ of the form $\left(X^{\prime}, m\right)$ with $X^{\prime} \cong X$.

Next we consider the variety

$$
\text { emat }_{\mathcal{K}, M}^{X}(\mathrm{k}):=\{(m, f) \in M(X, X) \times \mathcal{K}(X, X) \mid m f=f m\} .
$$

Re mark. In general emat ${ }_{\mathcal{K}, M}^{X}(\mathrm{k})$ is not irreducible, but it is connected. Indeed, consider the action of the multiplicative group $\mathrm{Gl}_{1}(\mathrm{k})$ on emat ${ }_{\mathcal{K}, M}^{X}(\mathrm{k})$ by scalar multiplication. Then we find that $(0,0)$ lies in the closure of any orbit and consequently $(0,0)$ lies in every irreducible component.

3.2. If $X \in \operatorname{Obj} \mathcal{K}$, we write $\#(X)$ for the number of direct summands of $X$.

Proposition. Let $(\mathcal{K}, M)$ be a bimodule problem, let $X_{1}, \ldots, X_{n}$ represent the isoclasses of indecomposable objects of $\mathcal{K}$ and $X_{0}:=\bigoplus_{i=1}^{n} X_{i}$. Then the following are equivalent:

(a) $(\mathcal{K}, M)$ is tame.

(b) $\operatorname{dim} \operatorname{emat}_{\mathcal{K}, M}^{X}(\mathrm{k}) \leq \operatorname{dim}^{\operatorname{Aut}_{X}}(\mathrm{k})+\#(X)$ for all $X \in \operatorname{Obj} \mathcal{K}$.

(c) $\operatorname{dim} \operatorname{emat}_{\mathcal{K}, M}^{X_{0}^{m}}(\mathrm{k}) \leq n^{2} \operatorname{dim}$ Aut $_{X_{0}}(\mathrm{k})+n m$ for all $m \in \mathbb{N}$.

Proof. (a) $\Rightarrow$ (b) is Lemma 2 below; (b) $\Rightarrow$ (c) is trivial. (c) $\Rightarrow$ (a) follows from Drozd's theorem and Lemma 1 below by trivial estimations.

Corollary. Let $\Lambda$ be a d-dimensional k-algebra and $M$ a $\Lambda$ - $\Lambda$-bimodule. Then the bimodule problem $(\mathcal{P}(\Lambda), \bar{M})$ is tame if and only if

$$
\operatorname{dim} \operatorname{emat}_{\mathcal{P}(\Lambda), \bar{M}}^{\Lambda^{m}}(\mathrm{k}) \leq d m^{2}+d m \quad \text { for all } m \in \mathbb{N} .
$$

P r o o f. Observe that every finitely generated indecomposable projective $\Lambda$-module is a direct summand of ${ }_{\Lambda} \Lambda \in \mathcal{P}(\Lambda)$. Moreover, we trivially have $\#(\Lambda) \leq d$. Now, Lemma 2 implies " $\Rightarrow$ ", while the other direction follows from Drozd's theorem and Lemma 1 by simple estimations. 
Lemma 1. With the above notations, suppose that $(\mathcal{K}, M)$ is wild. Then there exists $p \in \mathbb{N}$ such that

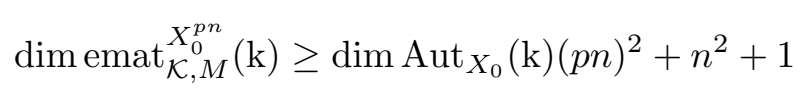

for all $n \in \mathbb{N}$.

Lemma 2. With the above notations, suppose that $(\mathcal{K}, M)$ is tame. Then

$$
\operatorname{dim} \operatorname{emat}_{\mathcal{K}, M}^{X}(\mathrm{k}) \leq \operatorname{dim} \operatorname{Aut}_{X}(\mathrm{k})+\#(X)
$$

for all objects $X$ in $\mathcal{K}$.

3.3. Proof of Lemma 1. Consider the affine variety $\bmod _{\mathrm{k}\langle x, y\rangle}^{n}(\mathrm{k})$ $:=\left(\operatorname{End}_{\mathrm{k}}\left(\mathrm{k}^{n}\right)\right)^{2}$ with the operation of $\mathrm{Gl}_{d}(\mathrm{k})$ by transport of structure, i.e. $\left(\mu_{x}, \mu_{y}\right)^{g}=\left(g^{-1} \mu_{x} g, g^{-1} \mu_{y} g\right)$; thus the $\mathrm{Gl}_{d}(\mathrm{k})$-orbits on $\bmod _{\mathrm{k}\langle x, y\rangle}^{n}(\mathrm{k})$ correspond bijectively to the isoclasses of $d$-dimensional $\mathrm{k}\langle x, y\rangle$-(right-)modules. The elements of the locally closed subset $U_{n} \subset \bmod _{\mathrm{k}\langle x, y\rangle}^{d}(\mathrm{k})$ are by definition of the form

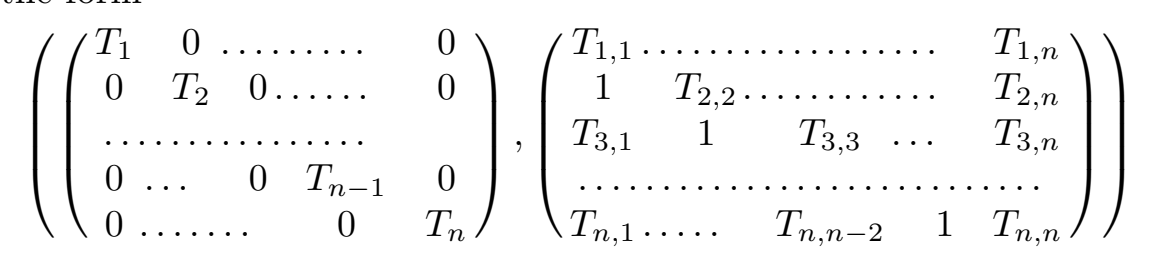

with $\prod_{i<j}\left(T_{i}-T_{j}\right) \neq 0$. The points of $U_{n}$ correspond to pairwise nonisomorphic $\mathrm{k}\langle x, y\rangle$-modules with trivial endomorphism ring.

Now, let $(X, \mathbf{b}, \varphi)$ be a $\mathrm{k}\langle x, y\rangle$-frame for $(\mathcal{K}, M)$ such that the induced functor $F_{X, \mathbf{b}, \varphi}$ preserves indecomposability and respects isoclasses $((\mathcal{K}, M)$ is wild!). Then $F_{X, \mathbf{b}, \varphi}$ gives rise to regular maps

$$
F_{X, \mathbf{b}, \boldsymbol{\varphi}}^{n}: \bmod _{\mathrm{k}\langle x, y\rangle}^{n}(\mathrm{k}) \rightarrow \operatorname{mat}_{\mathcal{K}, M}^{X \otimes \mathrm{k}^{n}}(\mathrm{k}), \quad\left(\mu_{x}, \mu_{y}\right) \mapsto \sum_{i=1}^{n} b_{i} \otimes \varphi_{i}\left(\mu_{x}, \mu_{y}\right) .
$$

Here, for example, $x y x\left(\mu_{x}, \mu_{y}\right):=\mu_{x} \mu_{y} \mu_{x}$. Notice that $F_{X, \mathbf{b}, \boldsymbol{\varphi}}$ maps different $\mathrm{Gl}_{n}(\mathrm{k})$-orbits to different $\mathrm{Aut}_{X \otimes \mathrm{k}^{n}}(\mathrm{k})$-orbits, thus the restriction to $U_{n}$ is injective.

Next, take $Y \in \operatorname{Obj} \mathcal{K}$ such that $X \oplus Y \cong X_{0}^{p}$ for some $p \in \mathbb{N}$ and consider the composition

$$
\widehat{F}_{X, \mathbf{b}, \varphi}: \bmod _{\mathrm{k}\langle x, y\rangle}^{n}(\mathrm{k}) \stackrel{F_{X, \mathbf{b}, \varphi}}{\longrightarrow} \operatorname{mat}_{\mathcal{K}, M}^{X \otimes \mathrm{k}^{n}}(\mathrm{k}) \stackrel{\mathrm{can}^{\prime}}{\longrightarrow} \operatorname{mat}_{\mathcal{K}, M}^{(X \oplus Y)}(\mathrm{k}) \otimes \mathrm{k}^{n} .
$$

Again, the restriction to $U_{n}$ is injective and the constructible subset $\left({ }^{1}\right)$ $Z_{n}:=\widehat{F}_{X, \mathbf{b}, \boldsymbol{\varphi}}\left(U_{n}\right) \subset \operatorname{mat}_{\mathcal{K}, M}^{(X \oplus Y)}(\mathrm{k}) \otimes \mathrm{k}^{n}$ intersects any $\operatorname{Aut}_{(X \oplus Y)^{n}}(\mathrm{k})$-orbit

$\left({ }^{1}\right)$ See $[12$, p. 91$]$ for the definition and basic properties of constructible sets; compare [13] and [14] for earlier use of this concept in representation theory. 
at most in one point. Standard calculations using $\operatorname{dim} Z_{n}=\operatorname{dim} U_{n}=n^{2}+1$ show finally

$$
\begin{aligned}
& \operatorname{dimemat}{ }_{\mathcal{K}, M}^{X_{0}^{p n}}(\mathrm{k})=\operatorname{dimemat}_{\mathcal{K}, M}^{(X \oplus Y)^{n}}(\mathrm{k}) \geq \operatorname{dim} \operatorname{Aut}_{(X \oplus Y)^{n}}(\mathrm{k})+n^{2}+1 \\
& =\operatorname{dim}_{\mathrm{Aut}_{X_{0}}(\mathrm{k})(p n)^{2}+n^{2}+1 .}
\end{aligned}
$$

3.4. Proof of Lemma 2. As in the case of varieties of modules (see [2], and also [6, Sect. 2], [15, 1.3]), using the fact that an object of $\operatorname{Mat}(\mathcal{K}, M)$ of the form $(X, m)$ has at most $\#(X)$ direct summands, we find in $\operatorname{mat}_{\mathcal{K}, M}^{X}(\mathrm{k})$ a constructible subset $C$ of dimension at most $\#(X)$ such that $C^{\operatorname{Aut}_{X}(\mathrm{k})}=\operatorname{mat}_{\mathcal{K}, M}^{X}(\mathrm{k})$.

On the other hand, we may stratify mat $_{\mathcal{K}, M}^{X}(\mathrm{k})$ into the locally closed subsets

$$
\operatorname{mat}_{\mathcal{K}, M}^{X, t}(\mathrm{k}):=\left\{m \in \operatorname{mat}_{\mathcal{K}, M}^{X}(\mathrm{k}) \mid \operatorname{dim} \operatorname{End}_{(\mathcal{K}, M)}(X, m)=t\right\} .
$$

Since $\operatorname{dim} C \leq \#(X)$ we get $\operatorname{dim} \operatorname{mat}_{\mathcal{K}, M}^{X, t}(\mathrm{k}) \leq\left(\operatorname{dim}_{A^{\prime}} \operatorname{ut}_{X}(\mathrm{k})-t\right)+\#(X)$.

Next we consider the locally closed subsets

$$
\operatorname{emat}_{\mathcal{K}, M}^{X, t}(\mathrm{k}):=\left\{(m, f) \in \operatorname{emat}_{\mathcal{K}, M}^{X}(\mathrm{k}) \mid m \in \operatorname{mat}_{\mathcal{K}, M}^{X, t}(\mathrm{k})\right\}
$$

of emat ${ }_{\mathcal{K}, M}^{X, t}(\mathrm{k})$ and observe that emat $\mathrm{K}_{\mathcal{K}, M}^{X, t}(\mathrm{k})$ is a vector bundle of rank $t$ over mat ${ }_{\mathcal{K}, M}^{X, t}(\mathrm{k})$ and therefore

$$
\operatorname{dim} \operatorname{emat}_{\mathcal{K}, M}^{X, t}(\mathrm{k}) \leq \operatorname{dim}_{\operatorname{Aut}_{X}}(\mathrm{k})+\#(X) \quad \forall t \in\left\{1, \ldots, \operatorname{dim}_{\operatorname{Aut}_{X}}(\mathrm{k})\right\},
$$

while for all other $t$ obviously emat ${ }_{\mathcal{K}, M}^{X, t}(\mathrm{k})=\emptyset$. This proves Lemma 2 .

3.5. In this subsection we concentrate on bimodule problems of the form $(\mathcal{P}(\Lambda), \bar{M})$.

Proposition. For given vector spaces $V, W$ and $n \in \mathbb{N}$ the function

$$
\delta_{n}: \operatorname{algb}_{V}^{W}(\mathrm{k}) \rightarrow \mathbb{N}, \quad(\lambda, \mu) \mapsto \operatorname{dimemat}_{\mathcal{P}(\Lambda), \bar{M}}^{\Lambda^{n}}(\mathrm{k}),
$$

is upper semicontinuous.

Proof. First, recall that for given $(\lambda, \mu) \in \operatorname{algb}_{V}^{W}(\mathrm{k})$ we write $(\Lambda, M)$ for the corresponding pair of an algebra and a bimodule with underlying vector spaces $(V, W)$. Now, $(\lambda, \mu)$ induces canonically on $V^{n \times n}$ the algebra structure $\lambda_{n}$ corresponding to $\operatorname{End}_{\Lambda}\left(\Lambda_{\Lambda} \Lambda^{n}\right)$ and on $W^{n \times n}$ the $\operatorname{End}_{\Lambda}\left(\Lambda_{\Lambda} \Lambda^{n}\right)$ bimodule structure $\mu_{n}$ corresponding to $\operatorname{Hom}_{\Lambda}\left(\Lambda^{n}, M \otimes_{\Lambda} \Lambda^{n}\right)$. With this setup we may consider the variety

$$
\begin{aligned}
& \operatorname{algbemat}_{V}^{W, n}(\mathrm{k}):=\left\{((\lambda, \mu), m, f) \in \operatorname{algb}_{V}^{W}(\mathrm{k}) \times W^{n \times n} \times V^{n \times n} \mid\right. \\
&\left.\mu_{n}(f \otimes m \otimes \mathrm{id})=\mu_{n}(\mathrm{id} \otimes m \otimes f)\right\}
\end{aligned}
$$

and the canonical projection $\pi_{n}$ : algbemat ${ }_{V}^{W, d}(\mathrm{k}) \rightarrow \operatorname{algb}_{V}^{W}(\mathrm{k})$. By construction we may identify the fibres $\pi_{n}^{-1}(\lambda, \mu)$ with emat $t_{\mathcal{P}(\Lambda), \bar{M}}^{\Lambda^{n}}(\mathrm{k})$. 
Now, the dimension of the fibres of $\pi_{n}$ is upper semicontinuous by Chevalley's theorem since $\pi_{n}$ admits the canonical section

$$
\sigma_{n}: \operatorname{algb}_{V}^{W}(\mathrm{k}) \rightarrow \operatorname{algbemat}_{V}^{W, n}(\mathrm{k}), \quad(\lambda, \mu) \mapsto((\lambda, \mu), 0,0),
$$

which meets any component of every fibre of $\pi_{n}$ (see Remark 3.1).

3.6. Proof of Theorem 2.4. Let the bimodule problem $\left(\mathcal{K}_{1}, M_{1}\right)$ be a deformation of the tame bimodule problem $\left(\mathcal{K}_{0}, M_{0}\right)$, and let $\left(\lambda_{i}, \mu_{i}\right) \in$ $\operatorname{algb}_{V}^{W}(\mathrm{k})(i=0,1)$ be the structure constants of the corresponding projective realizations. Thus we have, for all $m \in \mathbb{N}$,

$$
\operatorname{dim} \operatorname{emat}_{\mathcal{P}\left(\Lambda_{1}\right), \bar{M}_{1}}^{\Lambda_{1}^{m}}(\mathrm{k}) \leq \operatorname{dimemat} \mathrm{d}_{\mathcal{P}\left(\Lambda_{0}\right), \bar{M}_{0}}^{\Lambda_{m}^{m}}(\mathrm{k}) \leq[V: \mathrm{k}]\left(m^{2}+m\right) .
$$

Here, the first inequality comes from Proposition 3.5 (see our definition of deformations), while the second one is Corollary 3.2. On the other hand, this chain of inequalities shows the tameness of $\left(\mathcal{P}\left(\Lambda_{1}\right), \bar{M}_{1}\right)$ by the same corollary, and thus the tameness of $\left(\mathcal{K}_{1}, M_{1}\right)$.

\section{Examples}

4.1. The main application we have in mind is the case of vector space categories. Let us recall the definitions: If $\mathcal{K}$ is a Krull-Schmidt k-category together with a functor $|-|: \mathcal{K} \rightarrow \mathrm{k}$-mod, then the pair $(\mathcal{K},|-|)$ is called a vector space category. The subspace category $\mathcal{U}(\mathcal{K},|-|)$ has as objects the triples of the form $(X, V, \varphi)$ with $X \in \operatorname{Obj} \mathcal{K}, V \in \operatorname{Obj}(\mathrm{k}-m o d)$ and $\varphi \in \operatorname{Hom}_{\mathrm{k}}(V,|X|)$ with the obvious morphisms (see [17]-[19]). The category $\mathcal{U}(\mathcal{K},|-|)$ is canonically equivalent to the representations of the bimodule problem $\left(\underline{\mathcal{K}}, M_{|-|}\right)$, where $\underline{\mathcal{K}}:=\mathcal{K} \times \mathrm{k}-\bmod$ and

$$
M_{|-|}: \underline{\mathcal{K}}^{\mathrm{op}} \times \underline{\mathcal{K}} \rightarrow \mathrm{k}-\mathrm{mod}, \quad\left(\left(X_{1}, V_{1}\right),\left(X_{2}, V_{2}\right)\right) \mapsto \operatorname{Hom}_{\mathrm{k}}\left(V_{1},\left|X_{2}\right|\right) .
$$

The equivalence is given by

$$
\mathcal{U}(\mathcal{K},|-|) \rightarrow \operatorname{Mat}\left(\underline{\mathcal{K}}, M_{|-|}\right), \quad(X, V, \varphi) \mapsto((X, V), \varphi) .
$$

Thus we can speak of deformations of vector space categories, meaning deformations of the corresponding bimodule problem.

For applications the following setup is more handy: Recall from 2.3 that $\left(\underline{\mathcal{K}}, M_{|-|}\right)$is equivalent to $\left(\mathcal{P}(\underline{\Lambda}), \operatorname{Hom}_{\underline{\Lambda}}\left(-, \underline{M} \otimes_{\underline{\Lambda}}-\right)\right.$ by a standard construction. Now, observe that $\underline{\Lambda}=\Lambda \times \mathrm{k}$ with $\mathcal{K} \cong \mathcal{P}(\Lambda)$. Moreover, $\underline{M}$ inherits a natural $\mathrm{k}-\Lambda$-bimodule stucture, and we find a canonical isomorphism $\mathcal{U}(\mathcal{K},|-|) \cong \mathcal{U}\left(\mathcal{P}(\Lambda), \underline{M} \otimes_{\Lambda}-\right)$, the projective realization of $(\mathcal{K},|-|)$. This prompts us to study for given vector spaces $V$ and $W$ the variety algmat ${ }_{V}^{W}(\mathrm{k})$ which consists of the pairs $(\lambda, \mu) \in \operatorname{Hom}_{\mathrm{k}}(V \otimes V, V) \times \operatorname{Hom}_{\mathrm{k}}(W \otimes V, W)$, where $\lambda$ defines an associative unitary k-algebra $\Lambda$ on $V$, and $\mu$ defines a right $\Lambda$-module on $W$ with the natural action of $\mathrm{Gl}_{V}(\mathrm{k}) \times \mathrm{Gl}_{W}(\mathrm{k})$ by transport of structure. This gives rise to another obvious definition for deformations of 
vector space categories: $\left(\mathcal{K}_{1},|-|_{1}\right)$ is a deformation of $\left(\mathcal{K}_{0},|-|_{0}\right)$ if for the corresponding projective realizations $\left(\mathcal{P}\left(\Lambda_{i}\right), M_{i} \otimes_{\Lambda_{i}}-\right)$ etc. (compare 2.3).

Remark. Deformations in this latter sense are special cases of deformations with respect to the first definition. Indeed, there are natural embeddings $\operatorname{algmat}_{V}^{W}(\mathrm{k}) \rightarrow \operatorname{algb} V \oplus \mathrm{k} W$ and $\mathrm{Gl}_{V}(\mathrm{k}) \times \mathrm{Gl}_{W}(\mathrm{k}) \rightarrow \mathrm{Gl}_{V \oplus \mathrm{k}}(\mathrm{k}) \times$ $\mathrm{Gl}_{W}(\mathrm{k})$ compatible with the above constructions. This directly implies our statement.

Corollary. Deformations of tame vector space categories are tame.

4.2. Suppose for a moment that char $\mathrm{k} \neq 2$, and consider the algebraic family of vector space categories $\left(\mathcal{P}\left(\Lambda_{t}\right), \Lambda_{t} \otimes_{\Lambda_{t}}-\right)_{t \in \mathrm{k}}$, where $\Lambda_{t}=$ $\mathrm{k}[T] /\left(T^{2}+t T+t\right) \times \mathrm{k}[S] /\left(S^{2}+t S+t\right) \times \mathrm{k}$. Thus $\Lambda_{t} \cong \mathrm{k} \times \mathrm{k} \times \mathrm{k} \times \mathrm{k} \times \mathrm{k}$ for $t \neq 0$, while $\Lambda_{0}=\mathrm{k}[T] /\left(T^{2}\right) \times \mathrm{k}[S] /\left(S^{2}\right) \times \mathrm{k}$. The case $t \neq 0$ represents the five-subspace problem which is well known to be wild, and by the corollary above we conclude that also the case $t=0$ represents a wild problem.

4.3. Consider the following two algebras which we define by presenting their quivers with relations.

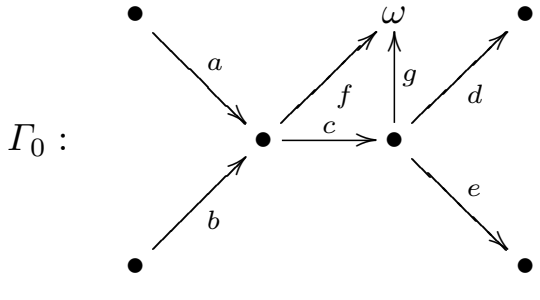

$0=a f=b f=c g$

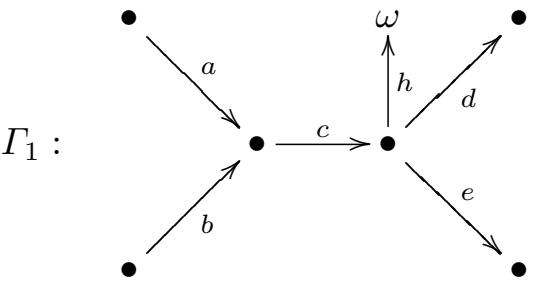

$0=a c h=b c h$

Then $\Gamma_{0}$-mod can be described by the representations of a clannish algebra [3]; in particular, $\Gamma_{0}$ is tame. $\Gamma_{1}$ is a deformation of $\Gamma_{0}$ and thus it is tame by [11]. We observe that both algebras are one-point extensions of a hereditary algebra $\Delta$ of type $\widetilde{\mathbb{D}}_{5}$ by regular modules, i.e. $\Gamma_{0}=\Delta\left[M_{0}\right]$ and $\Gamma_{1}=\Delta\left[M_{1}\right]$. Moreover, there exists an exact sequence of $\Delta$-modules

$$
0 \rightarrow \tau S \rightarrow M_{1} \rightarrow S \rightarrow 0,
$$

where $M_{0}=\tau S \oplus S$, and thus $M_{1}$ is a deformation of the semisimple $\Delta$ module $M_{0}$. Now let us consider the patterns (see [17] for definitions and conventions) for these one-point extensions:

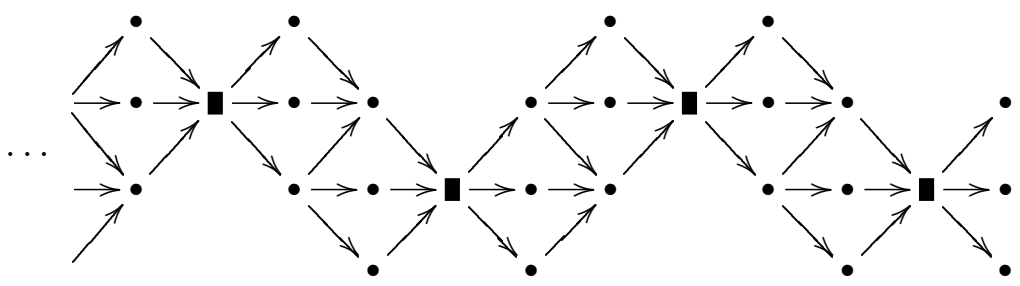




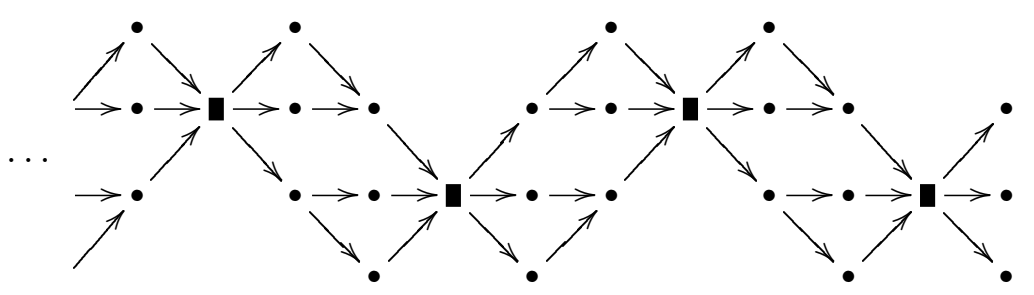

The corresponding vector space categories must be tame since we already know that the $\Gamma_{i}$ are tame. This is not new: indeed, these patterns appear already in Ringel's list of tame patterns.

If we take only finite pieces of these two patterns and consider the corresponding vector space categories, say $\left(\mathcal{K},|-|_{i}\right)$, we find that $\left(\mathcal{K},|-|_{1}\right)$ is a deformation of $\left(\mathcal{K},|-|_{0}\right)$. Here, $\mathcal{K}$ is the Krull-Schmidt category generated by a finite number of indecomposable $\Delta$-modules.

\section{References}

[1] K. Bongartz, On degenerations and extensions of finite-dimensional modules, Adv. in Math., to appear.

[2] W. W. Crawley-Boevey, On tame algebras and bocses, Proc. London Math. Soc. 56 (1988), 451-483.

[3] -, Functorial filtrations II: clans and the Gelfand problem, J. London Math. Soc. 40 (1989), 9-30.

[4] -, Matrix problems and Drozd's theorem, in: Topics in Algebra, Part 1: Rings and Representations of Algebras, Banach Center Publ. 26, PWN, Warszawa, 1990, 199-222.

[5] Yu. A. Drozd, Tame and wild matrix problems, in: Representation Theory II, Lecture Notes in Math. 832, Springer, 1980, 242-258.

[6] Yu. A. Drozd and G. M. Greuel, Tame-wild dichotomy for Cohen-Macaulay modules, Math. Ann. 294 (1992), 387-394.

[7] P. Gabriel, Finite representation type is open, in: Representations of Algebras, Lecture Notes in Math. 488, Springer, 1975, 132-155.

[8] P. Gabriel, L. A. Nazarova, A. V. Roiter, V. V. Sergejchuk and D. Vossieck, Tame and wild subspace problems, Ukrain. Math. J. 45 (1993), 313-352.

[9] P. Gabriel and A. V. Roiter, Representations of Finite-Dimensional Algebras, Encyclopedia of Math. Sci. 73, Algebra VIII, Springer, 1992.

[10] C. Geiß, Tame distributive algebras and related topics, Dissertation, Universität Bayreuth, 1993.

[11] —, On degenerations of tame and wild algebras, Arch. Math. (Basel) 64 (1995), $11-16$.

[12] R. Hartshorne, Algebraic Geometry, Springer, 1977.

[13] H. Kraft and C. Riedtmann, Geometry of representations of quivers, in: Representations of Algebras, London Math. Soc. Lecture Note Ser. 116, Cambridge Univ. Press, 1985, 109-145.

[14] J. A. de la Peña, On the dimension of module varieties of tame and wild algebras, Comm. Algebra 19 (1991), 1795-1805. 
[15] J. A. de la Peña, Functors preserving tameness, Fund. Math. 137 (1991), 77-185.

[16] J. A. de la Peña and D. Simson, Preinjective modules, reflection functors, quadratic forms, and Auslander-Reiten sequences, Trans. Amer. Math. Soc. 329 (1992), 733-753.

[17] C. M. Ringel, Tame algebras, in: Representation Theory I, Lecture Notes in Math. 831, Springer, 1980, 134-287.

[18] - Tame Algebras and Integral Quadratic Forms, Lecture Notes in Math. 1099, Springer, 1984.

[19] D. Simson, Linear Representations of Partially Ordered Sets and Vector Space Categories, Algebra Logic Appl. 4, Gordon and Breach, 1992.

Instituto de Matemáticas

Universidad Nacional Autonóma de México

04510 México, D.F., Mexico

E-mail: geiss@dgsca.unam.mx

Received 27 June 1995;

in revised form 27 November 1995 